\title{
Embeddings of orthogonal Grassmannians
}

\author{
Antonio Pasini
}

\begin{abstract}
In this paper I survey a number of recent results on projective and Veronesean embeddings of orthogonal Grassmannians and propose a few conjectures and problems.
\end{abstract}

Keywords : orthogonal polar spaces, grassmannians, Weyl modules, Veronese variety MSC 2000: 20C20, 20G15, 17B45, 17B50, 51E24, 14M15

\section{Organization of the paper}

This paper consists four sections, besides the present one. Section 2 is a survey of basic notions on projective and Veronesean embeddings and generating sets, to be freely used in the rest of the paper. In Section 3 we recall the definition of orthogonal Grassmannians and define two embeddings for each of them, called the Grassmann and Weyl embedding, respectively. In the special case of a dual polar space of type $B_{n}$ those two embeddings are Veronesean and one more Veronesean embedding can be defined, which we call the Veronese-spin embedding. In Section 4 we compare the embeddings defined in Section 3: the Veronese-spin embedding and the Weyl embedding of a dual polar space are always isomorphic while the Grassmann and Weyl embeddings are isomorphic when the underlying field has characteristic different from 2 . In the case of characteristic 2 things are more complicated. Most of Section 4 is devoted to a discussion of that case. Section 5 is devoted to universality. Nearly all results discussed in Sections 4 and 5 are taken from Cardinali and Pasini $[9,10,11]$. We will omit their proofs, referring the reader to $[9,10,11]$ for them, but we shall give short sketches of the proofs whenever it will be possible, so that the reader can get at least a flavor of the arguments used in them. 


\section{Basics on embeddings and generation}

Throughout this section $\Gamma=(\mathcal{P}, \mathcal{L})$ is a point-line geometry, $\mathcal{P}$ is its set of points and $\mathcal{L}$ its set of lines. We assume that the collinearity graph of $\Gamma$ is connected, that no two distinct lines of $\Gamma$ meet in more than one point and every line of $\Gamma$ has at least three points. The second condition is necessary for $\Gamma$ to admit a projective or laxly projective embedding while the third condition is necessary for the existence of a projective or Veronesean embedding. Connectedness is a sensible requirement. Anyway, I don't like disconnected objects.

\subsection{Projective embeddings}

Given $\Gamma$ as above, a projective embedding of $\Gamma$ in the projective space $\mathrm{PG}(V)$ of a vector space $V$ is an injective mapping $\varepsilon$ from the point-set $\mathcal{P}$ of $\Gamma$ to the set of points of $\mathrm{PG}(V)$ such that $\varepsilon$ maps every line of $\Gamma$ surjectively onto a line of $\mathrm{PG}(V)$ and $\varepsilon(\mathcal{P})$ spans $\mathrm{PG}(V)$.

Henceforth we will freely switch from $\mathrm{PG}(V)$ to $V$. In particular, we will commit the abuse of regarding $V$ instead of $\mathrm{PG}(V)$ as the codomain of $\varepsilon$, thus writing $\varepsilon: \Gamma \rightarrow V$ instead of $\varepsilon: \Gamma \rightarrow \mathrm{PG}(V)$. Accordingly, if $p \in \mathcal{P}$ we regard $\varepsilon(p)$ as a 1-dimensional subspace of $V$ and we take the dimension of $V$ as the dimension $\operatorname{dim}(\varepsilon)$ of $\varepsilon$.

If $\mathbb{F}$ is the underlying division ring of $V$ then we say that $\varepsilon$ is defined over $\mathbb{F}$, also that $\varepsilon$ is a projective $\mathbb{F}$-embedding for short. If all projective embeddings of $\Gamma$ are defined over the same division ring $\mathbb{F}$ (as it is the case for all geometries to be considered in this paper), then $\Gamma$ is said to be defined over $\mathbb{F}$.

Given two projective $\mathbb{F}$-embeddings $\varepsilon_{1}: \Gamma \rightarrow V_{1}$ and $\varepsilon_{2}: \Gamma \rightarrow V_{2}$, a morphism $f: \varepsilon_{1} \rightarrow \varepsilon_{2}$ from $\varepsilon_{1}$ to $\varepsilon_{2}$ is a semi-linear mapping $f: V_{1} \rightarrow V_{2}$ such that $\varepsilon_{2}=f \cdot \varepsilon_{1}$. Note that, since $\left\langle\varepsilon_{2}(\mathcal{P})\right\rangle=V_{2}$, the equality $\varepsilon_{2}=f \cdot \varepsilon_{1}$ forces $f: V_{1} \rightarrow V_{2}$ to be surjective. If $f$ is bijective then $f$ is called an isomorphism. When $\varepsilon_{1}$ and $\varepsilon_{2}$ are isomorphic we write $\varepsilon_{1} \cong \varepsilon_{2}$. Note that, if a morphism $f: \varepsilon_{1} \rightarrow \varepsilon_{2}$ exists then $f$ is uniquely determined by $\varepsilon_{1}$ and $\varepsilon_{2}$ modulo scalars (see e.g. Pasini and Van Maldeghem [23, Proposition 9]; we warn that the connectedness of $\Gamma$ is essential to obtain this result). If a morphism exists from $\varepsilon_{1}$ to $\varepsilon_{2}$ then we write $\varepsilon_{1} \geq \varepsilon_{2}$ and we say that $\varepsilon_{2}$ is a morphic image of $\varepsilon_{2}$. When $\varepsilon_{1} \geq \varepsilon_{2}$ but $\varepsilon_{1} \neq \varepsilon_{2}$ we write $\varepsilon_{1}>\varepsilon_{2}$.

Given an embedding $\varepsilon: \Gamma \rightarrow V$, let $K$ be a subspace of $V$ satisfying the following:

$(Q 1)$ if $x, y \in \mathcal{P}$ (possibly $x=y$ ) then $\langle\varepsilon(x), \varepsilon(y)\rangle \cap K=0$. In particular $K \cap \varepsilon(x)=0$ for every point $x \in \mathcal{P}$. 


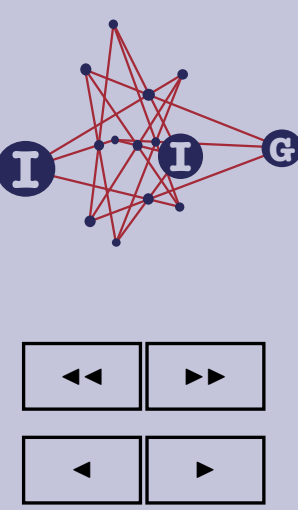

page $4 / 27$

go back

full screen

Given an embedding $\varepsilon: \Gamma \rightarrow V$ and an automorphism $g$ of $\Gamma$, a lifting of $g$ through $\varepsilon$ is a semi-linear mapping $\varepsilon(g): V \rightarrow V$ such that $\varepsilon(g) \cdot \varepsilon=\varepsilon \cdot g$. The lifting $\varepsilon(g)$ of $g$, if it exists, is uniquely determined modulo scalars. Clearly, it is invertible. Given a group $G$ acting on $\Gamma$ as a group of automorphisms, the embedding $\varepsilon$ is said to be $G$-homogeneous if for every $g \in G$ the automorphism of $\Gamma$ induced by $g$ lifts through $\varepsilon$ to a semi-linear map of $V$.

If $\varepsilon$ is absolutely universal then it is $\operatorname{Aut}(\Gamma)$-homogeneous. Let $\varepsilon_{1}$ and $\varepsilon_{2}$ be projective embeddings of $\Gamma$ and $f: \varepsilon_{1} \rightarrow \varepsilon_{2}$ a morphism. Suppose that $\varepsilon_{1}$ is $G$-homogeneous for some $G \leq \operatorname{Aut}(\Gamma)$ and $\operatorname{ker}(f)$ is stabilized by $G$. Then $\varepsilon_{2}$ is $G$-homogeneous.
\end{abstract}

close

quit

ACADEMIA

PRESS

NW

$\underline{\underline{\underline{11 \|}}}$

UNIVERSITEIT

GENT

\subsection{Veronesean embeddings}

Various definitions of Veronesean embeddings have appeared in the literature, sometimes under different names. The reader may see [27] for one of them. The underlying idea of each of those definitions is that lines are mapped onto conics, or even plane arcs, but that idea can be worked out in different ways, thus obtaining different definitions. We choose the following one.

Let $\Gamma=(\mathcal{P}, \mathcal{L})$ be a point line geometry satisfying the assumptions made at the beginning of this section. A Veronesean embedding of $\Gamma$ in (the projective space $\mathrm{PG}(V)$ of) a vector space $V$ defined over a commutative division ring (namely a field) $\mathbb{F}$ is an injective mapping $\varepsilon$ from the point-set $\mathcal{P}$ of $\Gamma$ to the set of points of $\mathrm{PG}(V)$ such that $\varepsilon$ maps every line of $\Gamma$ onto a non-singular conic of $\mathrm{PG}(V)$, for every line $l \in \mathcal{L}$ the projective plane spanned by the conic $\varepsilon(l)$ intersects the set $\varepsilon(\mathcal{P})$ just in $\varepsilon(l)$, and $\varepsilon(\mathcal{P})$ spans $\mathrm{PG}(V)$.

All definitions and conventions stated for projective embeddings in Subsection 2.1 can be rephrased for Veronesean embeddings word for word. In particular, we can still construct the hull $\tilde{\varepsilon}$ of $\varepsilon$ starting from $\left(\left\{V_{x}\right\}_{x \in \mathcal{P} \cup \mathcal{L}},\left\{\iota_{p, l}\right\}_{(p, l) \in \mathcal{F}}\right)$ where $V_{x}=\varepsilon(x)$ when $x \in \mathcal{P}$ and $V_{x}=\langle\varepsilon(x)\rangle=\left\langle\cup_{p \in x} V_{x}\right\rangle$ when $x \in \mathcal{L}$. As before, for every flag $(p, l) \in \mathcal{F}$ the mapping $\iota_{p, l}$ is the inclusion embedding of $V_{p}$ in $V_{l}$. Now $\varepsilon(l)=\{\varepsilon(p)\}_{p \in l}$ is a non-singular conic of the projective plane $\mathrm{PG}\left(V_{l}\right)$ while in the previous subsection $\mathrm{PG}\left(V_{l}\right)$ is a projective line and $\varepsilon(l)$ is its set of points. However this difference has no effect on the construction of $\widetilde{V}$ (see [22], where the hulls as defined here are called linear hulls).

We have claimed that all what is said in Subsection 2.1 for projective embeddings can be carried to Veronesean embeddings with nearly no modification. This is true, but when dealing with quotients more precise remarks are necessary. Indeed, certain situations can occur now that are not paralleled by anything occurring for projective embeddings.

Given a Veronesean embedding $\varepsilon: \Gamma \rightarrow V$, let $K$ be a subspace of $V$ satisfying 



\section{Embeddings of orthogonal Grassmannians}

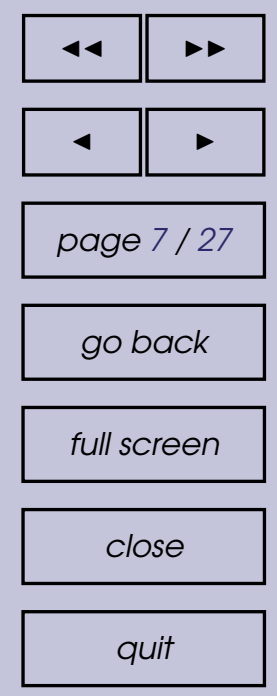

\subsection{Grassmannians of the building of type $B_{n}$ and their em- beddings}

For $n \geq 2$ let $V_{2 n+1} \cong V(2 n+1, \mathbb{F})$ be a $(2 n+1)$-dimensional vector space over a field $\mathbb{F}$ and $q$ a non-singular quadratic form of $V_{2 n+1}$ with Witt index $n \geq 2$. Let $\mathcal{B}_{n}$ the building of type $B_{n}$ associated to the pair $\left(V_{2 n+1}, q\right)$, where the elements of type $k=1,2, \ldots, n$ ( $k$-elements for short) are the $k$-dimensional subspaces of $V_{2 n+1}$ totally singular for $q$, with containment as the incidence relation. The building $\mathcal{B}_{n}$ is described by the following Dynkin diagram, where the integers written over the nodes of the diagram are the types:

$\left(B_{n}\right)$

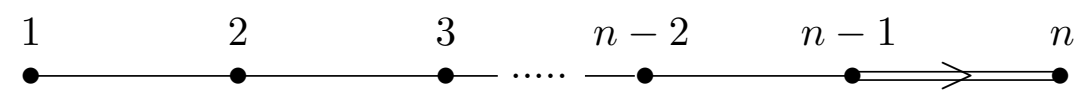

For $1 \leq k \leq n$, the $k$-shadow of a flag $F$ of $\mathcal{B}_{n}$ is the set of $k$-elements incident to $F$. The $k$-Grassmannian $\mathcal{B}_{n, k}$ of $\mathcal{B}_{n}$ is the point-line geometry defined as follows. The points of $\mathcal{B}_{n, k}$ are the $k$-elements of $\mathcal{B}_{n}$. When $1<k<n$ the lines of $\mathcal{B}_{n, k}$ are the $k$-shadows of the flags of $\mathcal{B}_{n}$ of type $\{k-1, k+1\}$. The geometry $\mathcal{B}_{n, 1}$ is the polar space associated to $\mathcal{B}_{n}$. Its lines are the 1 -shadows of the 2-elements of $\mathcal{B}_{n}$. The geometry $\mathcal{B}_{n, n}$ is usually regarded as the dual of the polar space $\mathcal{B}_{n, 1}$. Its lines are the $n$-shadows of the $(n-1)$-elements of $\mathcal{B}_{n}$. For such an element $X$, let $l_{X}$ be its $n$-shadow. Then

$$
l_{X}=\left\{Z \mid X \subset Z \subset X^{\perp}, \operatorname{dim}(Z)=n, Z \text { totally singular }\right\}
$$

where $X^{\perp}$ is the orthogonal of $X$ with respect to $q$. (Recall that $X$ is an $(n-1)$ dimensional totally singular subspace of $V_{2 n+1}$.) The vector space $X^{\perp} / X$ is 3-dimensional and $l_{X}$ is a non-singular conic in the projective plane $\mathrm{PG}\left(X^{\perp} / X\right)$.

Grassmann embeddings. For $1 \leq k \leq n$ let $W_{2 n+1, k}:=\wedge^{k} V_{2 n+1}$ and let $\varepsilon_{n, k}^{\mathrm{gr}}$ be the mapping from the set of points of $\mathcal{B}_{n, k}$ to the set of points of $\mathrm{PG}\left(W_{2 n+1, k}\right)$ defined by the following clause. Let $X$ be a $k$-element of $\mathcal{B}_{n}$ and $\left\{v_{1}, \ldots, v_{k}\right\}$ a basis of $X$ regarded as a subspace of $V_{2 n+1}$. The 1-dimensional subspace $\left\langle v_{1} \wedge \cdots \wedge v_{k}\right\rangle$ of $W_{2 n+1, k}$ does not depend on the choice of the basis $\left\{v_{1}, \ldots, v_{k}\right\}$ of $X$. We put $\varepsilon_{n, k}^{\mathrm{gr}}(X)=\left\langle v_{1} \wedge \cdots \wedge v_{k}\right\rangle$. It is easy to see (and well known) that this mapping is injective.

The mapping $\varepsilon_{n, 1}^{\mathrm{gr}}$ is just the natural embedding of the polar space $\mathcal{B}_{n, 1}$ in $V_{2 n+1}$. If $1<k<n$ then $\varepsilon_{n, k}^{\mathrm{gr}}$ is a projective embedding of $\mathcal{B}_{n, k}$ in the subspace $W_{2 n+1, k}^{\mathrm{gr}}:=\left\langle\varepsilon_{n, k}^{\mathrm{gr}}\left(\mathcal{B}_{n, k}\right)\right\rangle$ of $W_{2 n+1, k}$ spanned by $\varepsilon_{n, k}^{\mathrm{gr}}\left(\mathcal{B}_{n, k}\right)$ (where we take the 



\section{$1 \frac{1}{1}=0$
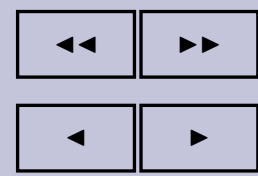 \\ page $10 / 27$ \\ go back \\ full screen \\ close \\ $\varepsilon_{n}^{\text {spin }}<\varepsilon_{n, n}^{\mathrm{Sp}}$, since $\operatorname{dim}\left(\varepsilon_{n, n}^{\mathrm{Sp}}\right)=\left(\begin{array}{c}2 n \\ n\end{array}\right)-\left(\begin{array}{c}2 n \\ n-2\end{array}\right)>2^{n}=\operatorname{dim}\left(\varepsilon_{n}^{\text {spin }}\right)$. When $2<|\mathbb{F}|$ the embedding $\varepsilon_{n, n}^{\mathrm{Sp}}$ is the hull of $\varepsilon_{n}^{\text {spin }}$ (Cooperstein [13] for the finite case and De Bruyn and Pasini [18] for the general case), hence it is universal. The case $\mathbb{F}=\mathbb{F}_{2}$ is exceptional. Indeed when $\mathbb{F}=\mathbb{F}_{2}$ the universal projective embedding of $\mathcal{B}_{n, n}$ has dimension equal to $\left(2^{n}+1\right)\left(2^{n-1}+1\right) / 3>\left(\begin{array}{c}2 n \\ n\end{array}\right)-\left(\begin{array}{c}2 n \\ n-2\end{array}\right)=\operatorname{dim}\left(\varepsilon_{n, n}^{\mathrm{Sp}}\right)$ (Li [21]; also Blokhuis and Brouwer [6]). \\ Finally, let $n=2$. We have $\varepsilon_{2,1}^{\mathrm{Sp}} \cong \varepsilon_{2}^{\mathrm{spin}}<\varepsilon_{2,2}^{\mathrm{Sp}} \cong \varepsilon_{2,1}^{\mathrm{gr}} \cong \varepsilon_{2,1}^{\mathrm{W}}$. \\ 3.2. Grassmannians of the building of type $D_{n}$ and their em- beddings}

quit

We denote by $\mathcal{D}_{n}$ the building of type $D_{n}$ defined over $\mathbb{F}$, where $n \geq 3$. Note that, given a non-singular quadratic form $q^{+}$of Witt index $n$ in $V_{2 n}=V(2 n, \mathbb{F})$, the non-trivial subspaces of $V_{2 n}$ totally singular for $q^{+}$, with their dimensions taken as types, form a non-thick building $\overline{\mathcal{D}}_{n}$ of Coxeter type $C_{n}$. The building $\mathcal{D}_{n}$ is obtained from $\overline{\mathcal{D}}_{n}$ by dropping the elements of type $n-1$ and partitioning the set of $n$-elements in two families, marked by two distinct types, say $(n, 0)$ and $(n, 1)$, where two elements $X$ and $Y$ of type $(n, 0)$ and $(n, 1)$ are declared to be incident precisely when $\operatorname{dim}(X \cap Y)=n-1$.

$\left(D_{n}\right)$

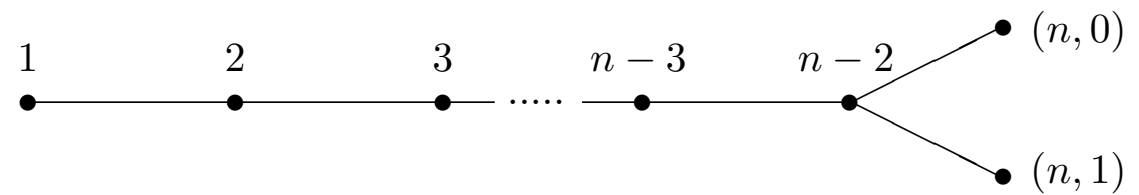

We allow $n=3$. Recall that the Coxeter diagram $D_{3}$ is the same as $A_{3}$, but with the usual types $1,2,3$ replaced with $(3,0), 1$ and $(3,1)$ respectively. In other words, $\mathcal{D}_{3}=\operatorname{PG}(3, \mathbb{F})$, the elements of $\mathcal{D}_{3}$ of type $1,(3,0)$ and $(3,1)$ being respectively the lines, the points and the planes of $\mathrm{PG}(3, \mathbb{F})$ (or lines, planes and points, if we prefer so).

For $k<n$, the $k$-Grassmannian $\overline{\mathcal{D}}_{n, k}$ of $\overline{\mathcal{D}}_{n}$ is defined just in the same way as the $k$-Grassmannian $\mathcal{B}_{n, k}$ of $\mathcal{B}_{n}$. We put $\mathcal{D}_{n, k}:=\overline{\mathcal{D}}_{n, k}$ and we call $\mathcal{D}_{n, k}$ the $k$-Grassmannian of $\mathcal{D}_{n}$.

The 1-Grassmannian $\mathcal{D}_{n, 1}$ of $\mathcal{D}_{n}$ is the polar space defined by $q^{+}$on $V_{2 n}$. Regarded $V_{2 n}$ as a hyperplane of $V_{2 n+1}=V(2 n+1, \mathbb{F})$, we can accordingly regard $\mathcal{D}_{n, 1}$ as a hyperplane of the polar space $\mathcal{B}_{n, 1}$ (which is defined in $V_{2 n+1}$ ). Similarly, $\mathcal{D}_{n, k}$ is the subgeometry induced by $\mathcal{B}_{n, k}$ on the set of $k$-subspaces of $V_{2 n}$.

Note that the points of $\mathcal{D}_{n, n-1}$ are the $\{(n, 0),(n, 1)\}$-flags of $\mathcal{D}_{n}$ while the lines of $\mathcal{D}_{n, n-1}$ correspond to flags of $\mathcal{D}_{n}$ of type $\{n-2,(n, 0)\}$ or $\{n-2,(n, 1)\}$. 



\subsection{A list of the embeddings defined in this section}

1. (Grassmann) $\varepsilon_{n, k}^{\mathrm{gr}}: \mathcal{B}_{k, n} \rightarrow W_{2 n+1, k}^{\mathrm{gr}} \subseteq W_{2 n+1, k}$. Projective when $k<n$ and Veronesean when $k=n$. It is a quotient of $\varepsilon_{n, k}^{\mathrm{W}}$ (item 4 of this list).

2. (Spin) $\varepsilon_{n}^{\text {spin }}: \mathcal{B}_{n, n} \rightarrow W_{n}^{\text {spin }}=V_{n}\left(\lambda_{n}\right)$. Projective. Dimension equal to $2^{n}$.

3. (Veronese-spin) $\varepsilon_{n}^{\mathrm{ver}}: \mathcal{B}_{n, n} \rightarrow W_{n}^{\mathrm{ver}} \subseteq V\left(\left(\begin{array}{c}2^{n}+1 \\ 2\end{array}\right), \mathbb{F}\right)$. Veronesean.

4. (Weyl) $\varepsilon_{n, k}^{\mathrm{W}}: \mathcal{B}_{k, n} \rightarrow V_{n}(\lambda)$, with $\lambda=\lambda_{k}$ for $k<n$ and $\lambda=2 \cdot \lambda_{n}$ when $k=n$. Projective when $k<n$ and Veronesean when $k=n$. In any case it has dimension equal to $\left(\begin{array}{c}2 n+1 \\ k\end{array}\right)$.

5. (Symplectic) $\varepsilon_{n, k}^{\mathrm{Sp}}: \mathcal{B}_{n, k} \rightarrow W_{2 n, k}^{\mathrm{Sp}}$. It only exists when $\mathbb{F}$ is a perfect field of characteristic 2. It is projective, with dimension equal to $\left(\begin{array}{c}2 n \\ k\end{array}\right)-\left(\begin{array}{c}2 n \\ k-2\end{array}\right)$.

6. (Grassmann) $\eta_{n, k}^{\mathrm{gr}}: \mathcal{D}_{k, n} \rightarrow W_{2 n, k}^{\mathrm{gr}} \subseteq W_{2 n, k}, k<n$. Projective. It is a quotient of $\eta_{n, k}^{\mathrm{W}}$ (see below).

7. (Weyl) $\eta_{n, k}^{\mathrm{W}}: \mathcal{D}_{k, n} \rightarrow V_{n}\left(\mu_{k}\right)$, where $k<n$ and $\mu_{n-1}=\mu_{n, 0}+\mu_{n, 1}$. Projective. Dimension equal to $\left(\begin{array}{c}2 n \\ k\end{array}\right)$.

\section{More on the previous embeddings}

\subsection{Grassmann and Weyl embeddings}

It is well known that $\varepsilon_{n, 1}^{\mathrm{gr}} \cong \varepsilon_{n, 1}^{\mathrm{W}}$ and $\eta_{n, 1}^{\mathrm{gr}} \cong \eta_{n, 1}^{\mathrm{W}}$ for any choice of the field $\mathbb{F}$. So, throughout this subsection we assume $k>1$.

Theorem 4.1. Let $\operatorname{char}(\mathbb{F}) \neq 2$. Then:

(1) $\varepsilon_{n, k}^{\mathrm{gr}} \cong \varepsilon_{n, k}^{\mathrm{W}}$ for every $k=2,3, \ldots, n$.

(2) $\eta_{n, k}^{\mathrm{gr}} \cong \eta_{n, k}^{\mathrm{W}}$ for every $k=2,3, \ldots, n-1$.

Sketch of the proof. Both (1) and (2) can be proved by exploiting the fact that the Weyl modules $V_{n}\left(\lambda_{2}\right), \ldots, V_{n}\left(\lambda_{n-1}\right), V_{n}\left(2 \cdot \lambda_{n}\right), V_{n}\left(\mu_{1}\right), \ldots, V_{n}\left(\mu_{n-2}\right)$ and $V_{n}\left(\mu_{n, 0}+\mu_{n, 1}\right)$ are irreducible when $\operatorname{char}(\mathbb{F}) \neq 2$, but in [9] we have used a different, more elementary argument to prove (1). The bulk of the proof given in [9] is to show that if $\operatorname{char}(\mathbb{F}) \neq 2$ then the set of points of $\mathcal{B}_{n, k}$ is a generating set for the $k$-Grassmannian $\mathcal{G}_{k}$ of $\mathrm{PG}\left(V_{2 n+1}\right) \cong \mathrm{PG}(2 n, \mathbb{F})$ in the sense of Subsection 2.3. Having proved this, let $\gamma_{k}$ be the natural embedding of $\mathcal{G}_{k}$ in $W_{2 n+1, k}$, mapping every $k$-subspace $\left\langle v_{1}, \ldots, v_{k}\right\rangle$ of $V_{2 n+1}$ onto $\left\langle v_{1} \wedge \cdots \wedge v_{k}\right\rangle$. Then $\gamma_{k}\left(\mathcal{G}_{k}\right)$ spans $W_{2 n+1, k}$. Moreover $\gamma_{k}$ induces $\varepsilon_{n, k}^{\mathrm{gr}}$ on $\mathcal{B}_{n, k}$. Therefore, 




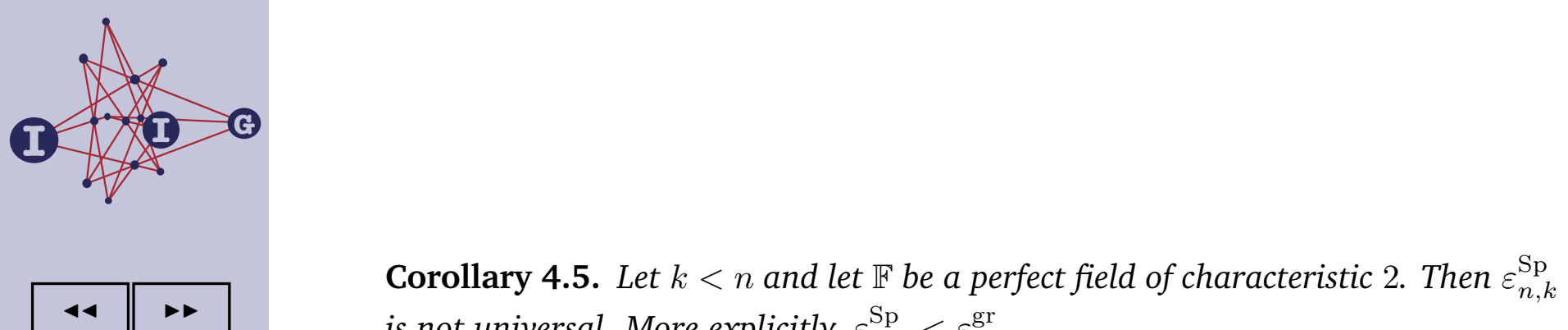
is not universal. More explicitly, $\varepsilon_{n, k}^{\mathrm{Sp}}<\varepsilon_{n, k}^{\mathrm{gr}}$.

Remark 4.6. The hypothesis that $k<n$ is essential for the conclusion of Corollary 4.5. Indeed, although $\varepsilon_{n, n}^{\mathrm{Sp}}$ is a proper quotient of $\varepsilon_{n, n}^{\mathrm{gr}}$, the latter is Veronesean rather than projective. So, the fact that $\varepsilon_{n, n}^{\mathrm{Sp}}$ is a quotient of $\varepsilon_{n, n}^{\mathrm{gr}}$ in the sense of Subsection 2.2 does not imply that $\varepsilon_{n, n}^{\mathrm{Sp}}$ is not universal. In fact, $\varepsilon_{n, n}^{\mathrm{Sp}}$ is universal when $2<|\mathbb{F}|<\infty$, as remarked at the end of Subsection 3.1.

full screen

close

Still assuming that $\operatorname{char}(\mathbb{F})=2$, we now turn to $\mathcal{D}_{n}$. With $q^{+}$as in Subsection 3.2, the sesquilinearization $\alpha$ of the quadratic form $q^{+}$is a non-degenerate alternating form of $V_{2 n}$. Let $\mathcal{C}_{n}$ be the building of type $C_{n}$ associated to $\alpha$. Then, for every $k=1,2, \ldots, n-1$, the $k$-Grassmannian $\mathcal{D}_{n, k}$ of $\mathcal{D}_{n}$ is a subspace (Subsection 2.3) of the $k$-Grassmannian $\mathcal{C}_{n, k}$ of $\mathcal{C}_{n}$. Let $\varepsilon_{n, k}^{\mathrm{Sp}}$ be the projective embedding of $\mathcal{C}_{n, k}$ mapping every $k$-subspace $\left\langle v_{1}, \ldots, v_{k}\right\rangle$ of $V_{2 n}$ totally isotropic for $\alpha$ onto the 1-dimensional subspace $\left\langle v_{1} \wedge \cdots \wedge v_{k}\right\rangle$ of $W_{2 n, k}=\wedge{ }^{k} V_{2 n}$ (compare the definition of symplectic embeddings in Subsection 3.1, but note that now $\mathcal{C}_{n}$ need not be isomorphic to $\mathcal{B}_{n}$, since $\mathbb{F}$ might be non-perfect). It is well known that $\varepsilon_{n, k}^{\mathrm{Sp}}$ is a projective embedding of $\mathcal{C}_{n, k}, \operatorname{dim}\left(\varepsilon_{n, k}^{\mathrm{Sp}}\right)=\left(\begin{array}{c}2 n \\ k\end{array}\right)-\left(\begin{array}{c}2 n \\ k-2\end{array}\right)$ and $\varepsilon_{n, k}^{\mathrm{Sp}}$ induces $\eta_{n, k}^{\mathrm{gr}}$ on $\mathcal{D}_{n, k}$. Therefore $\operatorname{dim}\left(\eta_{n, k}^{\mathrm{gr}}\right) \leq\left(\begin{array}{c}2 n \\ k\end{array}\right)-\left(\begin{array}{c}2 n \\ k-2\end{array}\right)$. Consequently, $\eta_{n, k}^{\mathrm{gr}}<\eta_{n, k}^{\mathrm{W}}$. A sharper statement is proved in [9], namely the following.

Theorem 4.7. Let $1<k<n$ and $\operatorname{char}(\mathbb{F})=2$. Then $\operatorname{dim}\left(\eta_{n, k}^{\mathrm{gr}}\right)=\left(\begin{array}{c}2 n \\ k\end{array}\right)-\left(\begin{array}{c}2 n \\ k-2\end{array}\right)$.

Sketch of the proof. Suppose firstly that $\mathbb{F}$ is perfect. Let $\mathcal{G}_{k}$ be the $k$-Grassmannian of $\operatorname{PG}\left(V_{2 n}\right)$. Then both $\mathcal{D}_{n, k}$ and $\mathcal{C}_{n, k}$ are subgeometries of $\mathcal{G}_{k}$. As $\mathcal{D}_{n, k} \subseteq \mathcal{C}_{n, k}$, we have $\left\langle\mathcal{D}_{n, k}\right\rangle_{\mathcal{G}_{k}} \subseteq\left\langle\mathcal{C}_{n, k}\right\rangle_{\mathcal{G}_{k}}$. The crucial step of the proof is to prove the reverse inclusion $\left\langle\mathcal{C}_{n, k}\right\rangle_{\mathcal{G}_{k}} \subseteq\left\langle\mathcal{D}_{n, k}\right\rangle_{\mathcal{G}_{k}}$. Having poved this, we obtain that $\left\langle\mathcal{D}_{n, k}\right\rangle_{\mathcal{G}_{k}}=\left\langle\mathcal{C}_{n, k}\right\rangle_{\mathcal{G}_{k}}$ and the statement of the theorem follows, recalling that the natural embedding of $\mathcal{G}_{k}$ in $W_{2 n, k}$ induces $\varepsilon_{n, k}^{\mathrm{Sp}}$ on $\mathcal{C}_{n, k}$ and $\eta_{n, k}^{\mathrm{gr}}$ on $\mathcal{D}_{n, k}$.

The inclusion $\left\langle\mathcal{C}_{n, k}\right\rangle_{\mathcal{G}_{k}} \subseteq\left\langle\mathcal{D}_{n, k}\right\rangle_{\mathcal{G}_{k}}$ is proved in [9] by exploiting the fact that, since $\mathcal{C}_{n} \cong \mathcal{B}_{n}$ (because $\mathbb{F}$ is assumed to be perfect) and $\mathcal{D}_{n, 1}$ is a hyperplane of $\mathcal{B}_{n, 1}$, the subgeometry $\mathcal{D}_{n, 1}$ is in fact a hyperplane of $\mathcal{C}_{n, 1}$. We refer the reader to [9] for the details of this proof.

When $\mathbb{F}$ is non-perfect the conclusion of the theorem can be obtained as in the proof of Theorem 4.2 , by descent to the prime subfield $\mathbb{F}_{2}$ of $\mathbb{F}$.

Remark 4.8. We have defined $\eta_{n, k}^{\mathrm{gr}}$ with the help of the Grassmann embedding $\varepsilon_{n, k}^{\mathrm{Sp}}$ of $\mathcal{C}_{n, k}$. It is worth remarking that $\varepsilon_{n, k}^{\mathrm{Sp}}$ is in fact a Weyl embedding (see e.g. Blok [1]). 


\subsection{The Veronese-spin embedding}

The next theorem is Theorem 1 of [10].

Theorem 4.9. We have $\varepsilon_{n}^{\mathrm{ver}} \cong \varepsilon_{n, n}^{\mathrm{W}}$ for every choice of the field $\mathbb{F}$.

Sketch of the proof. The group $\mathrm{SL}\left(2^{n}, \mathbb{F}\right)$ can be lifted from $V_{2 n}=V\left(2^{n}, \mathbb{F}\right)$ to $V\left(\left(\begin{array}{c}2^{n}+1 \\ 2\end{array}\right), \mathbb{F}\right)$ via the Veronesean quadratic map. Thus, $V\left(\left(\begin{array}{c}2^{n}+1 \\ 2\end{array}\right), \mathbb{F}\right)$ can be regarded as an $\mathrm{SL}\left(2^{n}, \mathbb{F}\right)$-module. One can prove that this module is isomorphic to the Weyl module $V\left(2 \cdot \omega_{1}\right)$ for $\operatorname{SL}\left(2^{n}, \mathbb{F}\right)$, where $\omega_{1}, \ldots, \omega_{2^{n}-1}$ are the fundamental dominant weights of the root system of type $A_{2^{n}-1}$. Next, regarding $V\left(2 \cdot \omega_{1}\right)$ as a $\operatorname{Spin}(2 n+1, \mathbb{F})$-module, as we can in view of the inclusion $\operatorname{Spin}(2 n+1, \mathbb{F}) \leq \mathrm{SL}\left(2^{n}, \mathbb{F}\right)$, we can recognize $V\left(2 \cdot \lambda_{n}\right)$ inside $V\left(2 \cdot \omega_{1}\right)$ as a $\operatorname{Spin}(2 n+1, \mathbb{F})$-submodule. Finally, it is proved that the isomorphism $V\left(2 \cdot \omega_{1}\right) \cong V\left(\left(\begin{array}{c}2^{n}+1 \\ 2\end{array}\right), F\right)$ induces an isomorphism from $V\left(2 \cdot \lambda_{n}\right)$ to $W_{n}^{\text {ver }}$.

Assumption. For the rest of this subsection we assume that $\operatorname{char}(\mathbb{F})=2$.

We firstly recall a number of known facts about the quadratic Veronesean map $\nu_{2^{n}}: V_{2^{n}} \rightarrow V\left(\left(\begin{array}{c}2^{n}+1 \\ 2\end{array}\right), \mathbb{F}\right)$. This map induces a Veronesean embedding of $\mathrm{PG}\left(2^{n}-1, \mathbb{F}\right)$ in $V\left(\left(\begin{array}{c}2^{n}+1 \\ 2\end{array}\right), \mathbb{F}\right)$, which we still denote by $\nu_{2^{n}}$. The image $\nu_{2^{n}}\left(\mathrm{PG}\left(2^{n}-1, \mathbb{F}\right)\right)$ of $\mathrm{PG}\left(2^{n}-1, \mathbb{F}\right)$ by $\nu_{2^{n}}$ is called a Veronesean variety. As noticed in the sketch of the proof of Theorem 4.9, the group $\mathrm{SL}\left(2^{n}, \mathbb{F}\right)$ lifts to $V\left(\left(\begin{array}{c}2^{n}+1 \\ 2\end{array}\right), \mathbb{F}\right)$. Clearly, it stabilizes the Veronesean variety $\nu_{2^{n}}\left(\mathrm{PG}\left(2^{n}-1, \mathbb{F}\right)\right)$. For every line $l$ of PG $\left(2^{n}-1, \mathbb{F}\right)$ let $n_{l}$ be the nucleus of the conic $\nu_{2^{n}}(l)$. The $n u-$ cleus subspace of $V\left(\left(\begin{array}{c}2^{n}+1 \\ 2\end{array}\right), \mathbb{F}\right)$ relative to $\nu_{2^{n}}$ is the subspace $\mathcal{N}$ of $V\left(\left(\begin{array}{c}2^{n}+1 \\ 2\end{array}\right), \mathbb{F}\right)$ spanned by the nuclei $n_{l}$, for $l$ a line of $\mathrm{PG}\left(2^{n}-1, \mathbb{F}\right.$ ) (Thas and Van Maldeghem [26]). The subspace $\mathcal{N}$ is stabilized by $\operatorname{SL}\left(2^{n}, \mathbb{F}\right)$ in its action on $V\left(\left(\begin{array}{c}2^{n}+1 \\ 2\end{array}\right), \mathbb{F}\right)$. Moreover, $\mathcal{N} \cap\left\langle\nu_{2^{n}}(l)\right\rangle=n_{l}$ for every line $l$ of $\mathrm{PG}\left(2^{n}-1, \mathbb{F}\right)$. Hence $\mathcal{N} \cap \nu_{2 n}(p)=$ 0 for every point $p$ of $\mathrm{PG}\left(2^{n-1}, \mathbb{F}\right)$.

Put $\mathcal{N}_{n, 0}^{\text {ver }}:=\mathcal{N}_{2^{n}} \cap W_{n}^{\text {ver }}$. For every $(n-1)$-element $X \in \mathcal{B}_{n, n-1}$ of $\mathcal{B}_{n}$, let $l_{X}$ be the line of $\mathcal{B}_{n, n}$ correspondig to $X$ and let $n_{X}$ be the nucleus of the conic $\varepsilon_{n}^{\mathrm{ver}}\left(l_{X}\right)=\nu_{2^{n}}\left(\varepsilon_{n}^{\mathrm{spin}}\left(l_{X}\right)\right)$. We put $\mathcal{N}_{n, 1}^{\mathrm{ver}}:=\left\langle n_{X}\right\rangle_{X \in \mathcal{B}_{n, n-1}}$.

Clearly, $\mathcal{N}_{n, 0}^{\mathrm{ver}} \supseteq \mathcal{N}_{n, 1}^{\mathrm{ver}}$ and both these subspaces are stabilized by the group $G=\operatorname{Spin}(2 n+1, \mathbb{F})$ in its action on $W_{n}^{\text {ver }}\left(\cong V\left(2 \cdot \lambda_{n}\right)\right.$ by Theorem 4.9). We can also define two mappings $\varepsilon_{n}^{\text {ver }} / \mathcal{N}_{n, 0}^{\text {ver }}$ and $\varepsilon_{n}^{\text {ver }} / \mathcal{N}_{n, 1}^{\text {ver }}$ from $\mathcal{B}_{n, n}$ to the set of 1-dimensional linear subspaces of $W_{n}^{\text {ver }} / \mathcal{N}_{n, 0}^{\text {ver }}$ and $W_{n}^{\text {ver }} / \mathcal{N}_{n, 1}^{\text {ver }}$ respectively and 


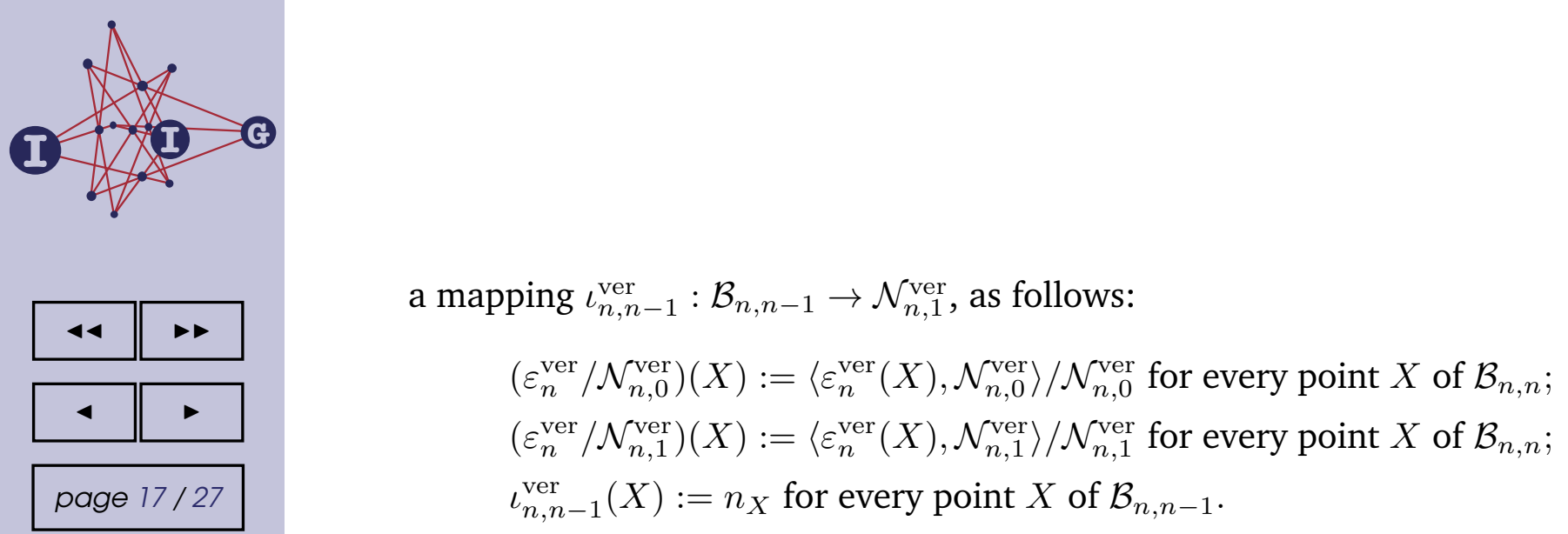

Lemma 4.10. Let $n=2$.

(1) Suppose that $\mathbb{F}$ is perfect. Then $\mathcal{N}_{2,0}^{\mathrm{ver}}$ defines a quotient of $\varepsilon_{2}^{\mathrm{ver}}$ and we have $\varepsilon_{n}^{\mathrm{ver}} / \mathcal{N}_{2,0}^{\mathrm{ver}} \cong \varepsilon_{2}^{\text {spin }}$.

(2) In any case, $\mathcal{N}_{2,1}^{\mathrm{ver}}$ defines a quotient of $\varepsilon_{2}^{\mathrm{ver}}$ and $\operatorname{dim}\left(\varepsilon_{2}^{\mathrm{ver}} / \mathcal{N}_{2,1}^{\mathrm{ver}}\right)=5$. If $\mathbb{F}$ is perfect then $\varepsilon^{\mathrm{ver}} / \mathcal{N}_{2,1}^{\mathrm{ver}} \cong \varepsilon_{2,2}^{\mathrm{sp}}$. If $\mathbb{F}$ is non-perfect then $\varepsilon^{\mathrm{ver}} / \mathcal{N}_{2,1}^{\mathrm{ver}}$ is laxly projective.

(3) $\iota_{2,1}^{\mathrm{ver}} \cong \varepsilon_{2,1}^{\mathrm{W}}\left(\cong \varepsilon_{2,1}^{\mathrm{gr}}\right)$.

Sketch of the proof. Claim (3) is Lemma 2 of [10]. It can be rephrased as follows: $\iota_{2,1}^{\text {ver }}\left(\mathcal{B}_{2,1}\right)$ is a copy of the quadric $\mathcal{B}_{2,1} \cong Q(4, \mathbb{F})$ in $\operatorname{PG}\left(\mathcal{N}_{2,1}^{\text {ver }}\right) \cong \operatorname{PG}(4, \mathbb{F})$ (notation as in Payne and Thas [24]). One of the points of $\operatorname{PG}\left(\mathcal{N}_{2,1}^{\mathrm{ver}}\right)$ is the nucleus of the quadric $\iota_{2,1}^{\mathrm{ver}}\left(\mathcal{B}_{2,1}\right)$. Both these claims admit straightforward proofs. (We warn that in [10] it is assumed that $\mathbb{F}$ is perfect, but this hypothesis plays no role in the proof of (3).) Moreover, denoted by $\mathcal{N}_{2,2}^{\mathrm{ver}}$ the nucleus of the quadric $\iota_{2,1}^{\mathrm{ver}}\left(\mathcal{B}_{2,1}\right)$, it is not difficult to see that $\mathcal{N}_{2,2}^{\mathrm{ver}}=\operatorname{ker}\left(\pi_{2,2}\right)$, where $\pi_{2,2}$ is the projection of $W_{2}^{\mathrm{ver}}=V_{2}\left(2 \lambda_{2}\right)$ onto $W_{2,2}^{\mathrm{gr}}$, as in Theorem 4.4. Accordingly, $\mathcal{N}_{2,1}^{\mathrm{ver}} / \mathcal{N}_{2,2}^{\mathrm{ver}}=\mathcal{N}_{2,2}^{\mathrm{gr}}$ (notation as in Theorem 4.2). Claim (2) now follows from Theorem 4.2.

Turning to (1), the crucial step in the proof of this claim is to prove that, for every line $l$ of $\mathcal{B}_{2,2}$, the intersection of $\mathcal{N}_{2,0}^{\mathrm{ver}}$ with the plane $\left\langle C_{l}\right\rangle$ spanned by the conic $C_{l}:=\varepsilon_{2}^{\mathrm{ver}}(l)$ is just the nucleus $n_{l}$ of $C_{l}$. This is proved in [10, Lemma 3.2] under the hypothesis that $\mathbb{F}$ is perfect, exploiting the fact that, when the underlying field is perfect, all lines through the nucleus of a conic are tangent to the conic. Having proved that $\mathcal{N}_{2,0}^{\mathrm{ver}} \cap\left\langle C_{l}\right\rangle=n_{l}$, it readily follows that $\mathcal{N}_{2,0}^{\mathrm{ver}}$ defines a quotient of $\varepsilon_{2}^{\mathrm{ver}}$ and $\varepsilon_{n}^{\mathrm{ver}} / \mathcal{N}_{2,0}^{\mathrm{ver}} \cong \varepsilon_{2}^{\mathrm{spin}}$.

So far we have defined $\mathcal{N}_{n, 0}^{\mathrm{ver}}, \mathcal{N}_{n, 1}^{\mathrm{ver}}$ and $\iota_{n, n-1}^{\mathrm{ver}}$. In the proof of Lemma 4.10 we have also defined $\mathcal{N}_{2,2}^{\mathrm{ver}}$ as the nucleus of the quadric $\iota_{2,1}^{\mathrm{ver}}\left(\mathcal{B}_{2,1}\right)$. This definition can be generalized as follows.

Let $n>2$. Given a $k$-element $X$ of $\mathcal{B}_{n}$ with $k \leq n-2$, the upper residue $\operatorname{Res}^{+}(X)$ of $X$ in $\mathcal{B}_{n}$ is a building of type $B_{n-k}$ with $\{k+1, \ldots, n\}$ as the set of types. We can define the $n$-Grassmannian $\operatorname{Res}_{n}^{+}(X)$ of $\operatorname{Res}^{+}(X)$ by taking the $n$-elements of $\operatorname{Res}^{+}(X)$ as points and the lines of $\mathcal{B}_{n, n}$ contained in $\operatorname{Res}^{+}(X)$ as lines. 







[10] $\_$Veronesean embeddings of dual polar spaces of orthogonal type. J. Combin. Theory Ser. A 120 (2013), 1328-1350.

[11] _ On certain submodules of Weyl modules for $\mathrm{SO}(2 n+1, \mathbb{F})$ with $\operatorname{char}(\mathbb{F})=2$. To appear in J. Group Theory.

[12] R. W. Carter, Lie Algebras of Finite and Affine Type, Cambridge Univ. Press, Cambridge, 2005.

[13] B. N. Cooperstein, On the generation of dual polar spaces of symplectic type over finite fields, J. Combin. Theory Ser. A 83 (1998), 221-232.

[14] _ Generating long root subgroup geometries of classical groups over finite prime fields, Bull. Belg. Math. Soc. Simon Stevin 5 (1998), 531-548.

[15] B. N. Cooperstein and E. E. Shult, Frames and bases of Lie incidence geometries. J. Geometry 60 (1997), 17-46.

[16] B. De Bruyn, The structure of the spin embedding of dual polar spaces and related geometries. European J. Combin. 29 (2008), 1242-1256.

[17] B. De Bruyn and A. Pasini, On symplectic polar spaces over non-perfect fields of characteristic 2, Linear Multilinear Algebra 57 (2009), 567-575.

[18] _ Generating symplectic and Hermitian dual polar spaces over arbitrary fields nonisomorphic to $\mathbb{F}_{2}$, Electronic Journal of Combinatorics 14 (2007), \#R54.

[19] J. E. Humphreys, Introduction to Lie Algebras and Representation Theory, Springer-Verlag, New York, 1972.

[20] A. Kasikova and E. E. Shult, Absolute embeddings of point-line geometries, J. Algebra 238 (2001), 265-291.

[21] P. Li, On the universal embedding of the $S p_{2 n}(2)$ dual polar space, $J$. Combin. Theory Ser. A 94 (2001), 100-117.

[22] A. Pasini, Embeddings and expansions, Bull. Belg. Math. Soc. Simon Stevin 10 (2003), 585-626.

[23] A. Pasini and H. Van Maldeghem, Some constructions and embeddings of the tilde geometry, Note di Matematica 21 (2002/2003), 1-33.

[24] S. E. Payne and J. A. Thas, Finite Generalized Quadrangles, Pitman, Boston, 1984. 
